\title{
Patterns of seizure control in patients with mesial temporal lobe epilepsy with and without hippocampus sclerosis
}

\author{
Padrões de controle de crises em pacientes com epilepsia de lobo temporal com ou sem \\ esclerose hipocampal \\ Ana Carolina Coan, Brunno M. Campos, Felipe P.G Bergo, Bruno Y. Kubota, Clarissa L. Yasuda, Marcia \\ E. Morita, Carlos A.M Guerreiro, Fernando Cendes
}

\begin{abstract}
Objective: Patients with mesial temporal lobe epilepsy (MTLE) may present unstable pattern of seizures. We aimed to evaluate the occurrence of relapse-remitting seizures in MTLE with (MTLE-HS) and without (MTLE-NL) hippocampal sclerosis. Method: We evaluated 172 patients with MTLE-HS (122) or MTLE-NL (50). Relapse-remitting pattern was defined as periods longer than two years of seizure-freedom intercalated with seizure recurrence. "Infrequent seizures" was considered as up to three seizures per year and "frequent seizures" as any period of seizures higher than that. Results: Thirty-seven (30\%) MTLE-HS and 18 (36\%) MTLE-NL patients had relapse-remitting pattern $\left(X^{2}, p=0.470\right)$. This was more common in those with infrequent seizures $\left(X^{2}, p<0.001\right)$. Twelve MTLE-HS and one MTLE-NL patients had prolonged seizure remission between the first and second decade of life $\left(X^{2}, p=0.06\right)$. Conclusion: Similar proportion of MTLE-HS or MTLE-NL patients present relapse-remitting seizures and this occurs more often in those with infrequent seizures.
\end{abstract}

Keywords: epilepsy, temporal lobe, seizure.

\section{RESUMO}

Objetivo: Pacientes com epilepsia do lobo temporal mesial (ELTM) podem apresentar padrão instável de crises epilépticas. Nosso objetivo foi avaliar ocorrência de crises remitente-recorrentes em ELTM com (ELTM-EH) e sem (ELTM-NL) esclerose hipocampal. Método: Avaliamos 172 pacientes com ELTM-EH (122) ou ELTM-NL (50). Padrão remitente-recorrente foi definido como períodos superiores a dois anos de remissão intercalados com recorrência de crises. Até três crises por ano foram consideradas como "infrequentes" e qualquer período com frequência maior como "frequentes". Resultados: Trinta e sete (30\%) pacientes com ELTM-EH e 18 (36\%) com ELTM-NL apresentaram crises remitente-recorrentes $\left(X^{2}, p=0,470\right)$, mais comum naqueles com crises infrequentes $\left(X^{2}, p<0,001\right)$. Doze pacientes com ELTM-EH e um ELTM-NL apresentaram remissão prolongada de crises entre a primeira e a segunda década de vida $\left(X^{2}, p=0,06\right)$. Conclusão: Proporção semelhante de pacientes com ELTM-EH e ELTM-NL apresentam crises remitente-recorrentes e isso ocorre com maior frequência em pacientes com crises esporádicas.

Palavras-chave: epilepsia, lobo temporal, convulsões.

Patients with epilepsy may present an unstable pattern of seizure control under antiepileptic drugs (AEDs) treatment, which may be inherent to the underlying pathophysiology of the disease.

Population-based studies demonstrate that nearly twothirds of patients with epilepsy will achieve seizure remission under AED treatment ${ }^{1}$. Although the majority of patients will have a stable pattern of response to the medications through long-term follow-ups, an intermittent pattern with refractory seizures interrupted by periods of remission can occur ${ }^{2}$. It has been estimated that around $16 \%$ of patients with epilepsies will have a relapse-remitting course, intercalating periods of seizure remission and refractory seizures ${ }^{3}$. It is also current knowledge that the underlying cause of the epilepsy is the major contributor to define AED response $e^{4}$. The role of possible different aetiologies to the occurrence of

Universidade Estadual de Campinas, Departamento de Neurologia, Laboratório de Neuroimagem, Campinas SP, Brazil.

Correspondence: Ana Carolina Coan; Universidade Estadual de Campinas, Departamento de Neurologia, Laboratório de Neuroimagem; Cidade Universitária Zeferino Vaz; 13083-970 Campinas SP, Brasil; E-mail: accoan@gmail.com

Conflict of interest: There is no conflict of interest to declare.

Support: São Paulo Research Foundation (FAPESP) funded this study, grants 2005/56578-4 and 2009/54552-9. Dr. Cendes received support from CNPq (Conselho Nacional de Desenvolvimento Científico e Tecnológico, Brazil).

Received 17 September 2014; Received in final form 23 September 2014; Accepted 14 October 2014. 
relapse-remitting patterns of seizure control has not been properly delineated.

Mesial temporal lobe epilepsy (MTLE) is the most common epileptic syndrome in adults and is most often associated with hippocampal sclerosis $(\mathrm{HS})^{5}$. The natural history of MTLE-HS has been extensively studied ${ }^{6,7}$. However, the natural history of MTLE with different underlying causes, especially MTLE in patients with normal MRI, is still not fully understood. Although relapse-remitting patterns of seizure control has been described in MTLE, its frequency in different studies is variable ${ }^{8}$ and the role of the presence of HS or other underlying pathology in this pattern of AED response has not been properly examined.

The objective of the present study was to evaluate and compare the patterns of seizure control across the lifespan of patients with MTLE with and without MRI signs of HS.

\section{METHOD}

\section{Patients' selection and classification}

We retrospectively evaluated the clinical data of 172 consecutive patients with clinical and electroencephalographic diagnosis of MTLE followed in a tertiary epilepsy centre (Epilepsy Clinic of Campinas State University).

All patients had either MTLE associated with HS (MTLEHS) or MTLE of unknown origin (MTLE with normal MRI, MTLE-NL). Patients with MTLE of other etiologies (tumor, vascular, cortical malformations) were not selected. All patients signed Informed Consent approved by the Ethics Committee of Universidade Estadual de Campinas prior to the acquisition of MRI.

For the definition of signs of HS, MRIs were acquired in a 3 Tesla Philips Intera Achieva scanner (Philips, Best, Netherlands) between August 2009 and June 2012. The MRI protocol included 3D-T1 weighted images (isotropic voxels of $1 \mathrm{~mm}$, acquired in the sagittal plane; $1 \mathrm{~mm}$ thick, flip angle $=8^{\circ}$, $\mathrm{TR}=7.0 \mathrm{~ms}, \mathrm{TE}=3.2 \mathrm{~ms}$, matrix $=240 \times 240, \mathrm{FOV}=240 \times 240$ ), T2 weighted image multi-eco coronal images $(3 \mathrm{~mm}$ thick, $\mathrm{TR}=3300 \mathrm{~ms}, \mathrm{TE}=30 / 60 / 90 / 120 / 150 \mathrm{~ms}$, matrix $=200 \times 180$, FOV $=180 \times 180$ ), as well as $3 \mathrm{~mm}$ thick coronal T1-inversion recovery and fluid attenuated inversion recovery (FLAIR) perpendicular to the long axis of hippocampus. HS signs were defined by MRI visual analysis plus MRI quantification of hippocampus volume and T2 signal, as described in a previous publication ${ }^{9}$. According to these analyses, patients were classified as MRI signs of HS (MTLE-HS, $\mathrm{N}=122$ ) or normal MRI (TLE-NL, $\mathrm{N}=50$ ).

\section{Clinical data and definitions}

The long-term seizure control pattern of each patient was assessed from the seizure onset period.
Patients were considered as relapse-remitting pattern of seizure control if they presented at least one period equal or longer than two years without any type of epileptic seizure intercalated with periods of seizure recurrence.

Independently from the classification of patients with or without relapse-remitting pattern, patients were divided in two groups according to the seizure frequency across the life spam. According to the frequency of seizures, patients were considered as having infrequent seizures if after achieving the adequate dose of the first AED they presented only simple partial seizures (SPS) or up to three complex partial seizures (CPS) per year and no generalized tonic-clonic seizures (GTCS). Patients with any period of seizures higher than that were considered as having frequent seizures.

\section{RESULTS}

There was no difference of sex or age distribution at the moment of the MRI acquisition between MTLE-HS and MTLE-NL patients. All patients had been under at least two different AEDs in optimized doses since the epilepsy onset. Detailed clinical information of the groups of patients is shown in Table.

Thirty-seven (30\%) MTLE-HS patients and 18 (36\%) MTLE-NL had a relapse-remitting pattern and no difference in the frequency of relapse-remitting pattern was observed between the groups $\left(X^{2}, \mathrm{p}=0.470\right)$.

In the MTLE-HS group, 104 (85\%) were classified as frequent seizures and 19\% (20/104) of those had relapseremitting pattern while $18(15 \%)$ were classified as infrequent seizures and $94 \%(17 / 18)$ of those had relapseremitting pattern. In MTLE-NL group, 38 (76\%) were classified as frequent seizures and $18 \%(7 / 38)$ of those had relapse-remitting pattern while $12(24 \%)$ were classified as infrequent seizures and $92 \%(11 / 12)$ of those had relapseremitting pattern. There was no difference in the distribution of the seizure frequency in the MTLE-HS and MTLE-NL groups $\left(X^{2}, \mathrm{p}=0.146\right)$. Among all patients with infrequent seizures, 93\% (28/30) had relapse-remitting pattern while it only occurred in 19\% (27/142) of patients with frequent seizures $\left(X^{2}, \mathrm{p}<0.001\right)$. Twelve (10\%) MTLE-HS patients had a prolonged period (more than five years) of seizure remission between the first and the second decade of life and it only occurred in only one patient with MTLE-NL and there was a trend in favour of patients with MTLE-HS for the occurrence of this pattern $\left(X^{2}, \mathrm{p}=0.06\right)$.

At the moment of the MRI acquisition, 13 (11\%) patients with MTLE-HS were free of seizures for a period equal or longer than two years (medium of three years of seizure-freedom, ranging from two to nine years). In this group, the medium time from the epilepsy onset to the present seizure remission was 22 years (ranging from three to 56 years). In 
MTLE-NL group, ten $(21 \%)$ were free of seizures for a period equal or longer than two years (medium of three years of seizure-freedom, ranging from two to six years). For MTLE-NL patients, the medium time from the epilepsy onset to the present seizure remission was 16 years (ranging from four to 38 years). There was no difference in the frequency of present seizure remission between MTLE-HS and MTLE-NL groups $\left(X^{2}, \mathrm{p}=0.102\right)$.

\section{DISCUSSION}

In this study, we evaluated the patterns of seizure control across the lifespan of patients with MTLE with and without MRI signs of HS and we observed that:

- A pattern of relapse-remitting seizures occurs in nearly one-third of patients with MTLE, independent of the presence of MRI signs of HS or normal MRI;

- In both MTLE-HS and MTLE-NL, the relapse-remitting pattern occurs more often in patients with infrequent seizures.

MTLE is not a single entity, but a group of different conditions that share common clinical and neurophysiologic characteristics $^{10}$. In MTLE, it is known that different aetiologies imply diverse long-term outcomes, with MTLE-HS being the subtype with the higher rates of drug-resistant seizures ${ }^{4}$. The knowledge of the natural history of the different types of MTLEs, including long-term response to AEDs, has important implications in the individual decisions for adequate choices of treatments. Moreover, it is also important for the understanding of the characteristics that lead different patients to infrequent or frequent seizures.

The ILAE consensus defines drug-resistant epilepsy as the failure of adequate trials of two tolerated and appropriately chosen AED schedules ${ }^{11}$. According to this definition, all patients included in the present study could be classified as refractory during one or different periods of their epilepsy evolution once all failed at least two different AEDs. Even those with current seizure remission tried more than two drugs before a later seizure control. However, it is known that epilepsies are dynamic diseases and the response to AEDs may vary throughout life without any external interference, possibly due to fluctuations inherent to its pathophysiology ${ }^{3}$.

In this study, we divided MTLE patients who had failed at least two different AEDs according to the seizure frequency they presented throughout their life span. We opted in this study for a restricted classification of patients and we considered the seizure frequency from the epilepsy onset. Only patients who never experienced more than three CPS per year and had no history of CTGS were classified as infrequent seizures. Although the definition of what are infrequent seizures is arbitrary and subjective, this dichotomy of patients with high or low seizure frequency is commonly seen in the clinical practice. Patients with infrequent seizures are not commonly submitted to epilepsy surgery due to the low morbidity of the SPS or the scarce CPS. The division of patients with the same epileptic syndrome but with infrequent or frequent seizures might help the better comprehension of the mechanisms of AED response.

An important aspect of our study is the patient sample. Although our patients were selected in a tertiary epilepsy centre, our service is composed by two different epilepsy clinics, one for surgical candidates and other for those who are not surgical candidates (non-surgical pathologies, patients who declined surgery and those with infrequent seizures or remission). Therefore, the complexity of the individuals in this study is possibly intermediate from what could be expected in a population-based study or a study conducted exclusively with patients of tertiary complexity.

In our study, we observed that nearly one-third of MTLE-HS and MTLE-NL patients present a pattern of relapse-remitting seizures and that the majority of patients with this pattern remained with a low frequency of seizure during the relapse periods. Three decades ago, Goodridge and Shorvon noted an intermittent pattern of AED response in which refractory seizures are interrupted by periods of remissions of at least two years in $12 \%$ of their patients ${ }^{2}$. Also, French et al. reported in a surgical series of adult patients with MTLE that a quarter had experienced previous periods of remission ${ }^{6}$. In our study, we observed higher rates of relapse-remitting pattern in both MTLE-HS and MTLE-NL. This variability of the frequency of relapseremitting patterns in different studies might be related to the definition and extent of the remission period or the complexity of the patients selected, which in our study was a more broad population of MTLE cases. More importantly, we observed that a relapse-remitting pattern in patients with MTLE and a high frequency of seizures during the relapse periods is not common and it is more often observed in those with low frequency of seizures.

We did not observe differences in the frequency of infrequent or frequent seizures between MTLE-HS and MTLE-NL patients. Equally, there was no significant difference of current seizure remission between MTLE-HS and MTLE-NL. This result differs from previous reports that observed higher rates of AED resistant seizures in MTLE-HS than in MTLE-NL ${ }^{12,13,14}$. In fact, in earlier studies, HS has been considered the epileptic pathology associated with the higher rates of patients with AED resistant seizures ${ }^{4}$. Although HS is classically associated with AED resistant seizures, in the last decades, with easier and more widespread access to MRI in non-surgical centres, it has been demonstrated that signs of HS can also be observed in individuals with infrequent seizures or seizure remission with or without 
Table. Clinical characteristics of patients with MTLE-HS and MTLE-NL.

\begin{tabular}{|c|c|c|c|}
\hline & MTLE-HS $(n=122)$ & MTLE-NL $(n=50)$ & $\mathrm{p}$ \\
\hline Sex & $77(63 \%)$ female 45 (37\%) male & $28(56 \%)$ female $22(44 \%)$ male & $X^{2}, p=0.385$ \\
\hline Median age at MRI (range) & 46 years $(17-73)$ & 43 years $(19-74)$ & Two-sample T-test, $p=0.251$ \\
\hline $\begin{array}{l}\text { Median number of AEDs } \\
\text { tried (range) }\end{array}$ & 5 AEDs (2-9) & 4 AEDs (2-9) & Two-sample T-test, $p=0.057$ \\
\hline Seizure onset (range) & 12 years $(0-50)$ & 16 years $(2-45)$ & Two-sample T-test, $p=0.003$ \\
\hline $\mathrm{FS} / \mathrm{IPI}$ & $13(11 \%) / 26(22 \%)$ & $3(6 \%) / 12(25 \%)$ & Fischer Exact Test, $p=0.402 / p=0.839$ \\
\hline
\end{tabular}

MTLE-HS: Mesial temporal lobe epilepsy with MRI signs of hippocampal sclerosis; MTLE-NL: Mesial temporal lobe epilepsy with normal MRI; n: Number of patients; AED: Anti-epileptic drug; FS: Febrile seizure; IPI: Initial precipitating injury.

$\mathrm{AEDs}^{15,16}$. However, the prevalence of HS in patients without refractory seizures remains unclear ${ }^{16}$. In our study, $15 \%$ of the patients with MTLE-HS were classified as having infrequent seizures and $11 \%$ were seizure-free for at least two years. This is an important finding because it demonstrates that in a more broad MTLE population, different from what is commonly seem in tertiary epilepsy centres, MTLE-HS do not have worse prognosis of seizure control than MTLE-NL. Population studies with early detection of signs of HS in patients with new onset epilepsies could demonstrate the accurate rates of AED resistance in MTLE-HS.

Although great improvement has been achieved in understanding the natural history of MTLEs in the last decades there is still a lack of adequate longitudinal data to confirm these findings. The difficulty to observe HS signs in children with MTLE is one of the problems to conduct longitudinal studies with MTLE-HS patients from the epilepsy onset. Other difficulty is the adequate definition of the epilepsy syndrome from the onset, which frequently demands investments in complex and costly complementary exams otherwise unnecessary for clinical treatment.

In conclusion, MTLE with or without MRI signs of HS share some characteristics of AED response, as similar rates of relapse-remitting pattern, which occurs more often in patients with low seizure frequency during the relapse periods.

\section{References}

1. Kwan P, Brodie MJ. Early identification of refractory epilepsy. N Engl J Med. 2010;342(5):314-9. http://dx.doi.org/10.1056/NEJM200002033420503

2. Goodridge DM, Shorvon SD. Epileptic seizures in a population of 6000. II: Treatment and prognosis. Br Med J. (Clin Res Ed). 1983;287(6393):645-7. http://dx.doi.org/10.1136/bmj.287.6393.645

3. Brodie MJ, Barry SJE, Bamagous GA, Norrie JD, Kwan P. Patterns of treatment response in newly diagnosed epilepsy. Neurology. 2012;78(20):1548-54. http://dx.doi.org/10.1212/WNL.0b013e3182563b19

4. Semah F, Picot MC, Adam C, Broglin D, Arzimanoglou A, Bazin B et al. Is the underlying cause of epilepsy a major prognostic factor for recurrence? Neurology. 1998;51(5):1256-62. http://dx.doi.org/10.1212/ WNL.51.5.1256

5. Hauser WA, Annegers JF, Kurland LT. Prevalence of epilepsy in Rochester, Minnesota: 1940-1980. Epilepsia. 1991;32(4):429-45. http://dx.doi.org/10.1111/j.1528-1157.1991.tb04675.x

6. French JA, Williamson PD, Thadani VM, Darcey TM, Mattson RH, Spencer SS et al. Characteristics of medial temporal lobe epilepsy: I. Results of history and physical examination. Ann Neurol. 1993;34(6):774-80. http://dx.doi.org/10.1002/ana.410340604

7. Berg AT. The natural history of mesial temporal lobe epilepsy. Curr Opin Neurol. 2008;21(2):173-8. http://dx.doi.org/10.1097/WC0.0b01 3e3282f36ccd

8. Sillanpää M, Schmidt D. Natural history of treated childhood-onset epilepsy: prospective, long-term population based study. Brain. 2006;129(Pt 3):617-24. http://dx.doi.org/10.1093/brain/awh726

9. Coan AC, Kubota BY, Bergo FPG, Campos BM, Cendes F. 3T MRI quantification of hippocampal volume and signal in mesial temporal lobe epilepsy improves detection of hippocampal sclerosis. AJNR Am J Neuroradiol .2014;35(1):77-83. http://dx.doi.org/10.3174/ajnr.A3640
10. Wieser HG, ILAE Commission on Neurosurgery of Epilepsy. Mesial temporal lobe epilepsy with hippocampal sclerosis. Epilepsia. 2004;45(6):695-714. http://dx.doi.org/10.1111/j.0013-9580.2004. 09004.x

11. Kwan P, Arzimanoglou A, Berg AT, Brodie MJ, Allen Hauser W, Mathern $G$ et al. Definition of drug resistant epilepsy: consensus proposal by the ad hoc Task Force of the ILAE Commission on Therapeutic Strategies. Epilepsia. 2010;51(6):1069-77. http://dx.doi. org/10.1111/j.1528-1167.2009.02397.x

12. Van Paesschen W, Connelly A, King MD, Jackson GD, Duncan JS. The spectrum of hippocampal sclerosis: a quantitative magnetic resonance imaging study. Ann Neurol. 1997;41(1):41-51. http://dx.doi. org/10.1002/ana.410410109

13. Cohen-Gadol AA, Bradley CC, Williamson A, Kim JH, Westerveld M, Duckrow RB et al. Normal magnetic resonance imaging and medial temporal lobe epilepsy: the clinical syndrome of paradoxical temporal lobe epilepsy. J Neurosur. 2005;102(5):902-9. http://dx.doi. org/10.3171/jns.2005.102.5.0902

14. Pittau F, Bisulli F, Mai R, Fares JE, Vignatelli L, Labate A et al. Prognostic factors in patients with mesial temporal lobe epilepsy. Epilepsia. 2009;50(Suppl s1):41-4. http://dx.doi.org/10.1111/j.15281167.2008.01969.x

15. Kobayashi E, Lopes-Cendes I, Guerreiro CA, Sousa SC, Guerreiro MM, Cendes F. Seizure outcome and hippocampal atrophy in familial mesial temporal lobe epilepsy. Neurology. 2001;56(2):166-72. http://dx.doi.org/10.1212/WNL.56.2.166

16. Labate A, Gambardella A, Andermann E, Aguglia U, Cendes F, Berkovic $\mathrm{SF}$ et al. Benign mesial temporal lobe epilepsy. Nat Rev Neurol. 2011;7(4):237-40. http://dx.doi.org/10.1038/nrneurol.2010.212 